\title{
Mechanism-based risk assessment strategy for drug-induced cholestasis using the transcriptional benchmark dose derived by toxicogenomics
}

\author{
Taisuke Kawamoto, Yuichi Ito, Osamu Morita and Hiroshi Honda
}

Safety Science Research, Kao Corporation, 2606 Akabane, Ichikaimachi, Haga, Tochigi 321-3497, Japan

(Received October 31, 2016; Accepted April 17, 2017)

\begin{abstract}
Cholestasis is one of the major causes of drug-induced liver injury (DILI), which can result in withdrawal of approved drugs from the market. Early identification of cholestatic drugs is difficult due to the complex mechanisms involved. In order to develop a strategy for mechanism-based risk assessment of cholestatic drugs, we analyzed gene expression data obtained from the livers of rats that had been orally administered with 12 known cholestatic compounds repeatedly for 28 days at three dose levels. Qualitative analyses were performed using two statistical approaches (hierarchical clustering and principle component analysis), in addition to pathway analysis. The transcriptional benchmark dose (tBMD) and tBMD 95\% lower limit (tBMDL) were used for quantitative analyses, which revealed three compound sub-groups that produced different types of differential gene expression; these groups of genes were mainly involved in inflammation, cholesterol biosynthesis, and oxidative stress. Furthermore, the tBMDL values for each test compound were in good agreement with the relevant no observed adverse effect level. These results indicate that our novel strategy for drug safety evaluation using mechanismbased classification and tBMDL would facilitate the application of toxicogenomics for risk assessment of cholestatic DILI.
\end{abstract}

Key words: Toxicogenomics, Microarray, tBMD, Cholestasis, Mechanism-based

\section{INTRODUCTION}

Drug-induced liver injury (DILI) has led to the withdrawal of many drugs from the market. Wang et al. (2013) reported that $44 \%$ of these were withdrawn due to severe DILI, such as acute hepatitis, fatty liver, and cholestasis. Cholestasis is a condition where cytotoxic bile acids accumulate (Björnsson and Olsson, 2005) and this accounts for approximately $20 \%$ of DILI in the USA and Denmark (Padda et al., 2011). In the diagnosis of DILI, cholestasis is widely evaluated clinically by measuring the levels of aspartate transaminase (AST), alanine aminotransferase (ALT), alkaline phosphatase (ALP), bilirubin, and $\gamma$-glutamyltransferase (GGT) (Ozer et al., 2008). In animal safety assessment experiments, biochemical measures of ALP, GGT, and bilirubin are utilized, in addition to histopathological findings such as bile duct injury (Fujii, 1997). However, the detection sensitivity of standard rodent evaluations of cholestatic chemicals is currently inadequate (Olson et al., 2000). In particular, his- topathological examination often underestimates the risk of DILI.

During drug development, the inhibitory effects on bile acid transporters such as the bile salt export pump (BSEP) are also evaluated. The clear correlation between inhibition of these transporters by some drugs and the incidence of cholestasis in both rats and humans (Fattinger et al., 2001; Funk et al., 2001) has led to the development of some in vitro assessment methods that focus on BSEP inhibition (Ogimura et al., 2011). However, not every BSEP inhibitor causes cholestasis (Morgan et al., 2010) and it is therefore necessary to consider other mechanisms during these evaluations. In fact, research findings have indicated that cholestasis can be induced via several mechanisms; these include inhibition of cell-cell junctions, inhibition of microfilament activity, and even inflammation (Vinken et al., 2013). For these reasons, the development of a mechanism-based and versatile method is required for the precise evaluation of the propensity of a drug to cause cholestasis.

Correspondence: Hiroshi Honda (E-mail: honda.hiroshi@kao.co.jp) 
To address these difficulties, some research has focused on toxicogenomics using cDNA microarrays. This approach has been referred by pharmaceutical fields (Boverhof and Zacharewski, 2006) as well as some public authorities (National Research Council, 2007; Chepelev et al., 2015; Organisation for Economic Co-operation and Development, 2016; Scientific Committee on Consumer Safety NfG, 2016). Toxicogenomics has the potential to detect key abnormalities and to improve understanding of biological mechanisms (Liu et al., 2017). However, the current toxicogenomics methods have two crucial features that limit their practical application.

First, despite recent intense attempts to detect cholestasis using machine-learning based on toxicogenomics, the sensitivity was limited (67\%) (Van den Hof et al., 2014a). The use of toxicogenomics with machine-learning analysis sometimes leads to "over-fitting", where the sensitivity during the training stage tends to decrease during validation using a new dataset. For example, Gusenleitner et al. (2014) conducted cross-validation analysis by machine learning using two toxicogenomics databases. However, this approach did not achieve the required level of sensitivity. Such an over-fitting problem might be caused by data heterogeneity, since several mechanisms of toxicity with different gene expression changes may underlie an adverse effect. Therefore, discriminating different gene expression patterns based on the mechanism of action would improve the sensitivity of prediction models. Indeed, Matsumoto et al. (2014) performed mechanism-based classification before the development of discriminators used to identify carcinogens; this approach resulted in the identification of high-performance discriminators in cross-validation using an external database. Thus, mechanism classification would be one promising approach to addressing over-fitting.

Second, the vast majority of current toxicogenomics methods involve qualitative approaches to mechanismbased classification. In contrast, quantitative methodology has been used less frequently for risk assessment. Yang et al. (2007) focused on dose-response effects using gene expression data and developed the transcriptional benchmark dose (tBMD) method; this was derived by applying the benchmark dose method to the estimation of transcriptional Point of Departure (tPoD) in cDNA microarray data. This tBMD approach has subsequently been used for the risk assessment of some substances (Clewell et al., 2014; Dong et al., 2016) and these studies found good agreement between tPoD and apical Point of Departure $(\mathrm{aPoD})$, a measure that is traditionally used, such as the no observed adverse effect level (NOAEL). Therefore, the combination of mechanism-based classification with the tBMD method could provide novel insights that would enable the pragmatic use of toxicogenomics for DILI risk assessment.

The present study aimed to develop a mechanism-based qualitative and quantitative methodology for the pragmatic safety assessment of cholestatic DILI. We employed the tBMD approach to quantitative risk assessment, followed by principal component analysis (PCA) and hierarchical clustering to analyze the mechanisms underlying cholestasis.

\section{MATERIALS AND METHODS}

\section{Data collection}

Clinical information about cholestatic chemicals was obtained from the Liver Tox database (US National Library of Medicine; http://livertox.nlm.nih.gov/index. html). The rat 28-day repeat dose tests in the Open TGGATEs (http://toxico.nibiohn.go.jp) (Igarashi et al., 2015) were reviewed to determine the PoD for each cholestatic chemical. Canonical diagnostic markers (blood ALP, GTP, and bilirubin) were used as endpoints for cholestasis. If significant cholestatic marker up-regulation was observed, the maximum dose that did not cause up-regulation of these markers was defined as the cholestatic NOAEL. On the other hand, if no significant up-regulation of cholestatic markers was observed, the maximum dose was defined as NOAEL without cholestatic markers. These NOAEL were termed the aPoD. Statistical results of cholestatic marker levels were used as previously reported by National Institutes of Biomedical Innovation, Health and Nutrition ("Histopathology photograph collection”, http://toxico.nibiohn.go.jp/english/seika.html).

\section{Qualitative analysis 1: mechanism classification}

Data from microarrays performed using Affymetrix Rat Genome 2302.0 chips (Santa Clara, CA, USA) were extracted from the Toxicogenomics Project-Genomics Assisted Toxicity Evaluation system (TG-GATEs). The microarray data obtained from the livers of rats treated with only high doses of 12 cholestatic chemicals (Table 1) for 28 days were pre-processed by MAS5 (Gautier et al., 2004) and then converted to logarithmic (base 2) fold changes $\left(\log _{2} \mathrm{Fc}\right)$ versus the solvent control group in order to identify differentially expressed genes. The differentially expressed genes were filtered by fold change $\left(\log _{2} \mathrm{Fc}>1\right)$ and Welch's $t$-test $(\mathrm{p}<0.05)$ and they were used in the subsequent steps if the differentially expressed genes were identified in over one-third of these 12 samples.

After extracting the differentially expressed genes, mechanism classification was performed by conduct- 
Mechanism-based quantitative toxicogenomic analysis of cholestasis

Table 1. Test compounds and experimental conditions.

\begin{tabular}{|c|c|c|c|c|c|c|}
\hline \multirow{2}{*}{ Name } & \multirow{2}{*}{ Abbreviation } & \multirow{2}{*}{ Vehicle } & \multirow{2}{*}{ Route } & \multicolumn{3}{|c|}{ Dose (mg/kg) } \\
\hline & & & & Low & Mid & High \\
\hline Chlorpromazine & $\mathrm{CPZ}$ & $0.5 \%$ methylcellulose & Gavage & 4.5 & 15 & 45 \\
\hline Cyclosporine A & $\mathrm{CSA}$ & corn oil & Gavage & 10 & 30 & 100 \\
\hline Diclofenac & $\mathrm{DFNa}$ & $0.5 \%$ methylcellulose & Gavage & 1 & 3 & 10 \\
\hline Erythromycin ethylsuccinate & EME & $0.5 \%$ methylcellulose & Gavage & 100 & 300 & 1000 \\
\hline Flutamide & FT & corn oil & Gavage & 15 & 50 & 150 \\
\hline Imipramine & IMI & $0.5 \%$ methylcellulose & Gavage & 10 & 30 & 100 \\
\hline Ketoconazole & $\mathrm{KC}$ & $0.5 \%$ methylcellulose & Gavage & 10 & 30 & 100 \\
\hline Methyltestosterone & MTS & $0.5 \%$ methylcellulose & Gavage & 30 & 100 & 300 \\
\hline Naphthyl isothiocyanate & ANIT & corn oil & Gavage & 1.5 & 5 & 15 \\
\hline Phenylbutazone & $\mathrm{PhB}$ & $0.5 \%$ methylcellulose & Gavage & 20 & 60 & 200 \\
\hline Sulindac & SUL & $0.5 \%$ methylcellulose & Gavage & 5 & 15 & 50 \\
\hline Tamoxifen & TMX & corn oil & Gavage & 6 & 20 & 60 \\
\hline
\end{tabular}

ing cluster analysis and PCA using R (Ver. 3.1.2, https:// cran.r-project.org/) and its packages (cluster analysis: pvclust; PCA: prcomp, rgl). Hierarchical clustering was performed using the pvclust method (Suzuki and Shimodaira, 2006). Ward's method was used and the cluster distance was based on Euclidean distance. Statistical significance was considered when $p$-values were $<0.05$.

\section{Qualitative analysis 2: pathway analysis}

The rank product method (Hong et al., 2006) and Welch's $t$-test were used for a secondary differentially expressed gene extraction within the individual clusters identified by Qualitative analysis 1 . The false discovery rate (FDR) for the rank product method and the p-value for the comparison between the control and treated rats in each classification were calculated (Welch's $t$-test). The significance thresholds applied were FDR $<0.001$ (rank product), $\mathrm{p}<0.01$ (Welch's $t$-test), and $\log _{2} \mathrm{Fc}>$ 0.58 . These secondary differentially expressed genes (Supplemental Table 1) are referred to below as cholestatic differentially expressed genes.

The canonical pathway analysis and upstream regulator analysis (Krämer et al., 2014) in Ingenuity Pathway Analysis (IPA; QIAGEN, North Rhine-Westphalia, Germany) were conducted using the cholestatic differentially expressed genes. The statistical significance threshold for the canonical pathway analysis was a $-\log$ p-value $>2$ (corresponding to $p<0.01$ ). The directions of the effects on molecules predicted to be upstream of the differentially expressed genes (activation versus inhibition) in the upstream regulator analysis could be predicted by the Z-score. A Z-score $>1.96$ was considered as the threshold of statistical significance.

\section{Quantitative analysis: derivation of $\mathrm{tPoD}$ and comparison with aPoD}

For the quantitative assessment of cholestatic effects using toxicogenomics, we analyzed microarray data obtained using three doses of these 12 cholestatic chemicals (low, middle, and high; Table 1). tBMD and the tBMD 95\% lower limit (tBMDL) of each gene in the presence of each chemical were calculated by BMDexpress (Yang et al., 2007). In short, the differentially expressed genes of each chemical (control, low, middle, and high doses) were imported to BMDexpress as $\log _{2} \mathrm{Fc}$ using the default settings. tBMD and tBMDL were then calculated for each cholestatic differentially expressed gene, excluding a set of 43 genes that showed differential expression for all or some treatments and were therefore regarded as non-specific. For model selection from Hill, power, linear, and polynomial, goodness-of-fit was cut off at $p>0.1$. Cholestatic differentially expressed genes were omitted if tBMD/tBMDL was $>10$ or if the lowest dose/BMDL was $>100$. Furthermore, the median values of tBMD and tBMDL in the presence of each chemical were calculated as $\mathrm{tPoD}$, for comparison with the aPoD. A scatter plot was used to analyze the correlation between tPoD and aPoD. A paired $t$-test was used to analyze the statistical significance of any differences between the tPoD and aPoD values.

\section{RESULTS}

\section{Cholestatic chemical selection}

Nine known cholestatic compounds were identified using the Liver Tox database: chlorpromazine (CPZ); cyclosporine A (CSA); diclofenac (DFNa); flutamide (FT); imipramine (IMI); ketoconazole (KC); methyltestosterone (MTS); sulindac (SUL); and tamoxifen (TMX). 
In addition, erythromycin ethylsuccinate (EME); naphthyl isothiocyanate (ANIT); and phenylbutazone (PhB) were also selected because these were previously reported to be cholestatic (Benjamin et al., 1981; Diehl et al., 1984; Ferreira et al., 2003). The present study thus investigated 12 cholestatic chemicals. Table 1 summarizes these and the in vivo experimental conditions.

\section{Qualitative classification of 12 cholestatic chemicals into three groups associated with inflammation, cholesterol biosynthesis, and oxidative stress}

Cluster analysis initially identified two statistically significant clusters (Fig. 1A). Cluster 1 included DFNa and SUL, while cluster 2 included the other test compounds. Cluster 2 contained two minor clusters that did not differ significantly. However, PCA clarified this sub-division (Fig. 1B) by separating MTS, TMX, PhB, IMI, CPZ, KC, and EME from FT, CSA, and ANIT. These analyses classified the 12 test compounds into three groups: Group 1, DFNa and SUL; Group 2, ANIT, FT, and CSA; and Group 3, MTS, TMX, CPZ, KC, EME, PhB, and IMI.

Next, secondary cholestatic differentially expressed genes (69 probe sets in Group 1,87 probe sets in Group 2, and 89 probe sets in Group 3) were extracted using the rank product method and Welch's $t$-test, followed by pathway analysis and upstream regulator analysis.

(A)

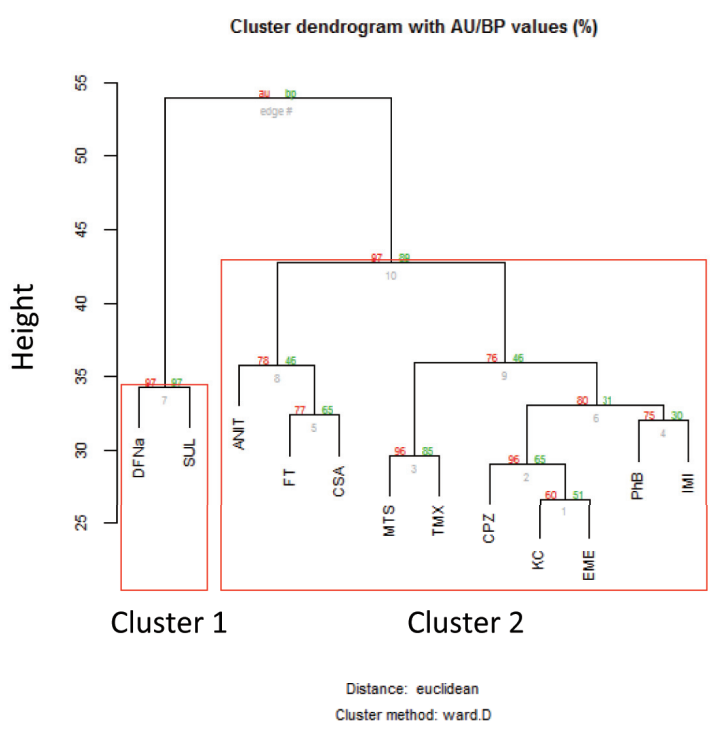

Fig. 2 shows the heat map for each cholestatic differentially expressed gene and Fig. 3 shows each pathway network and upstream regulators. In Group 1, inflammatory and/or immune responsive cytokines (S100a8/9, Cxcl1, Il-7) and signal mediators (Jak3, Stat3) were upregulated. In Group 2, genes associated with the oxidative stress response (Gpx2, Nqo1) were identified in the upstream regulator analysis, and an apoptosis-related gene (Hspala) was also up-regulated. Both of these profiles indicated cell damage, via different mechanisms. In Group 3, phase I/II metabolic enzymes such as Cyp2b1/2, Cyp4a8, Aldh1b1, Abcb1a/b, and Gstm3 were identified as differentially expressed genes, and nuclear receptors (the constitutive androstane receptor [CAR (NR1I3)] and the pregnane $\mathrm{X}$ receptor [PXR (NR1I2)]) were predicted as upstream regulators.

A summary of the canonical pathway analysis is shown in Table 2. Consistent with the upstream regulator analysis results, each chemical group was associated with specific pathways. For example, Group 1 effects were indicative of an inflammatory/damage response (role of IL-17A in psoriasis, role of JAK1 and JAK3 in $\gamma c$ cytokine signaling, acute phase response signaling, IL-9 signaling, coagulation system, IL-6 signaling), Group 2 effects related to cholesterol synthesis (cholesterol biosynthesis I, cholesterol biosynthesis II [via 24,25-dihydrolanosterol], cholesterol biosynthesis III [via desmosterol]), and Group 3
(B)

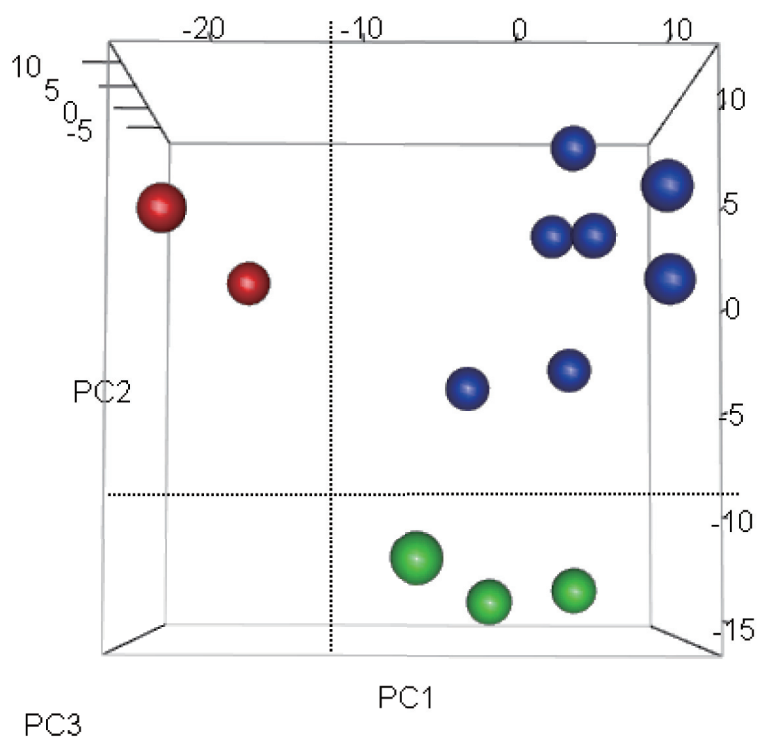

Fig. 1. Cholestatic mechanism using two unsupervised methods. (A) Cluster analysis of 12 chemicals. The red boxes enclose compounds that did not differ significantly within the groups. (B) PCA of 12 chemicals. Each color represents one group: red, Group 1 (DFNa, SUL); green, Group 2 (ANIT, FT, CSA); blue, Group 3 (MTS, TMX, CPZ, KC, EME, PhB, IMI). 
Mechanism-based quantitative toxicogenomic analysis of cholestasis

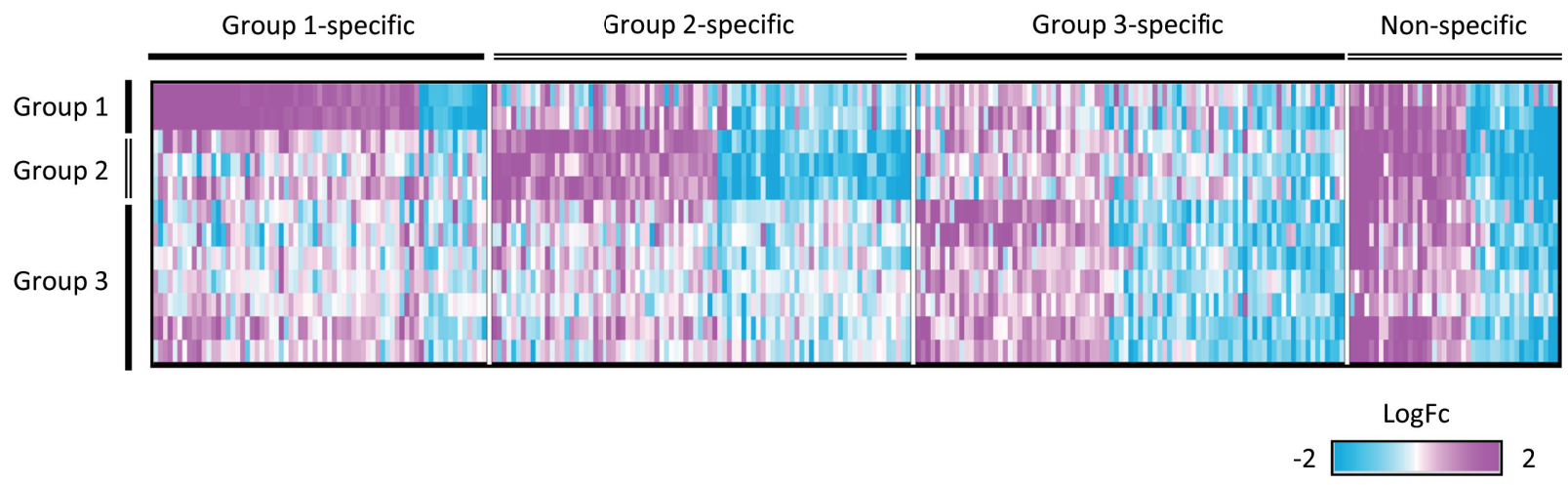

Fig. 2. Heat map of differentially expressed gene values for cholestatic chemicals. Each color represents the fold-change in relation to the solvent control. Some differentially expressed genes were specific for a group, while others were non-specific.

(A)

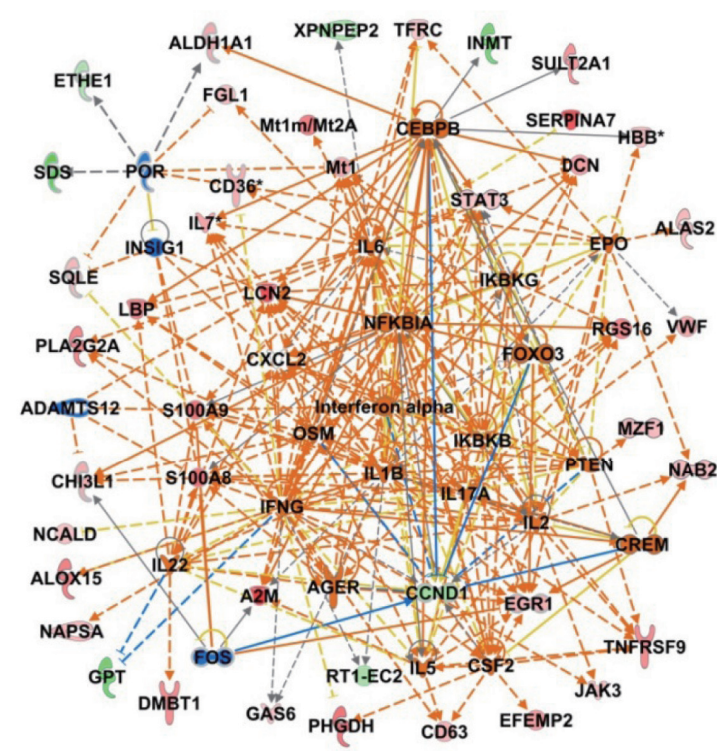

(B)

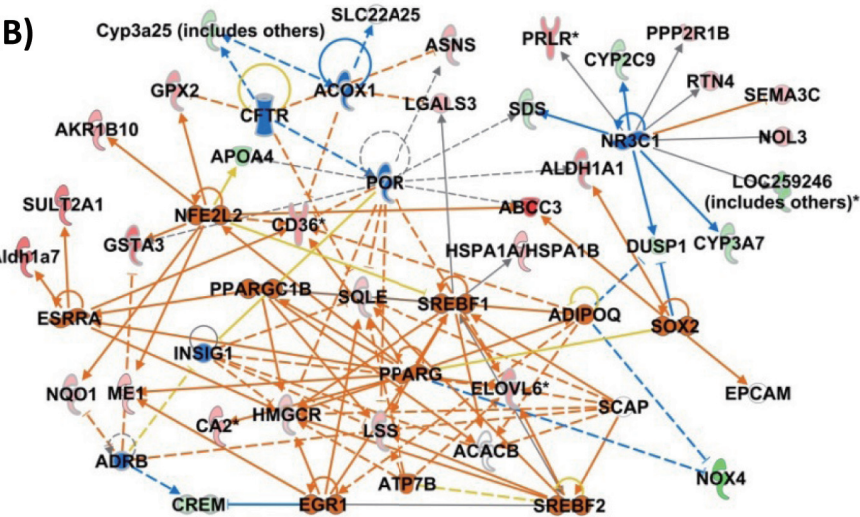

(C)

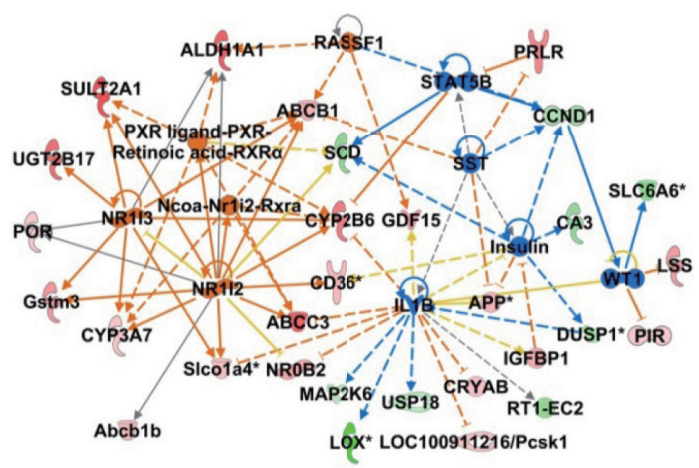

Fig. 3. Pathway analysis of three groups of cholestatic chemicals. The networks between differentially expressed genes and upstream regulators were generated using upstream regulator analysis. (A) Pathway network for Group 1. (B) Pathway network for Group 2. (C) Pathway network for Group 3. Red indicates up-regulated genes, green indicates down-regulated genes, orange indicates activated upstream-regulator molecules, and blue indicates inactivated upstream-regulator molecules. The color intensity indicates the extent of the change in level or activity. 
Table 2. Summary of the canonical pathway for each classified group.

\begin{tabular}{|c|c|c|c|c|}
\hline \multicolumn{2}{|c|}{ Canonical pathway } & Group 1 & Group 2 & Group 3 \\
\hline \multirow{14}{*}{$\begin{array}{l}\text { Group 1- } \\
\text { specific }\end{array}$} & Oncostatin M Signaling & 3.84 & 0 & 0 \\
\hline & Atherosclerosis Signaling & 3.28 & 1.02 & 1.03 \\
\hline & ErbB2-ErbB3 Signaling & 3.17 & 0.67 & 1.64 \\
\hline & Role of IL-17A in Psoriasis & 3.17 & 0 & 0 \\
\hline & Role of JAK1 and JAK3 in $\gamma c$ Cytokine Signaling & 3.06 & 0 & 0 \\
\hline & Acute Phase Response Signaling & 2.78 & 0 & 0.3 \\
\hline & Pancreatic Adenocarcinoma Signaling & 2.4 & 0 & 0.45 \\
\hline & IL-9 Signaling & 2.33 & 0 & 0 \\
\hline & Coagulation System & 2.3 & 0 & 0 \\
\hline & IL-6 Signaling & 2.29 & 0 & 1.08 \\
\hline & Alanine Biosynthesis II & 2.22 & 0 & 0 \\
\hline & Alanine Degradation III & 2.22 & 0 & 0 \\
\hline & iNOS Signaling & 2.11 & 0 & 0 \\
\hline & 1-serine Degradation & 2.05 & 1.9 & 0 \\
\hline \multirow{6}{*}{$\begin{array}{l}\text { Group 2- } \\
\text { specific }\end{array}$} & Superpathway of Cholesterol Biosynthesis & 1.09 & 3.67 & 0.96 \\
\hline & Cholesterol Biosynthesis I & 1.42 & 2.89 & 1.28 \\
\hline & Cholesterol Biosynthesis II (via 24,25-dihydrolanosterol) & 1.42 & 2.89 & 1.28 \\
\hline & Cholesterol Biosynthesis III (via Desmosterol) & 1.42 & 2.89 & 1.28 \\
\hline & Leukotriene Biosynthesis & 0 & 2.76 & 0 \\
\hline & Asparagine Biosynthesis I & 0 & 2.38 & 0 \\
\hline \multirow{8}{*}{$\begin{array}{l}\text { Group 3- } \\
\text { specific }\end{array}$} & Noradrenaline and Adrenaline Degradation & 0.97 & 1.96 & 3.29 \\
\hline & Estrogen Biosynthesis & 0 & 1.96 & 3.29 \\
\hline & PI3K/AKT Signaling & 1.28 & 0.39 & 2.77 \\
\hline & GADD45 Signaling & 1.25 & 0 & 2.56 \\
\hline & Glutathione-mediated Detoxification & 0 & 0.94 & 2.2 \\
\hline & NRF2-mediated Oxidative Stress Response & 0.38 & 1.4 & 2.18 \\
\hline & Cell Cycle Regulation by BTG Family Proteins & 1 & 0.87 & 2.04 \\
\hline & Aryl Hydrocarbon Receptor Signaling & 1.18 & 1.68 & 4.61 \\
\hline \multirow{2}{*}{$\begin{array}{l}\text { Groups } 1 / 2 \\
\text { merged }\end{array}$} & LXR/RXR Activation & 2.24 & 2.78 & 1.86 \\
\hline & Epoxysqualene Biosynthesis & 2.22 & 2.08 & 0 \\
\hline \multirow{20}{*}{$\begin{array}{l}\text { Groups } 2 / 3 \\
\text { merged }\end{array}$} & Xenobiotic Metabolism Signaling & 0.71 & 7.76 & 11.25 \\
\hline & PXR/RXR Activation & 1.77 & 5.09 & 11.17 \\
\hline & LPS/IL-1-Mediated Inhibition of RXR Function & 1.54 & 3.5 & 7.62 \\
\hline & Histamine Degradation & 1.3 & 2.65 & 4.36 \\
\hline & Oxidative Ethanol Degradation III & 1.25 & 2.55 & 4.2 \\
\hline & Nicotine Degradation III & 0 & 2.83 & 4.14 \\
\hline & Fatty Acid $\alpha$-oxidation & 1.23 & 2.51 & 4.14 \\
\hline & Putrescine Degradation III & 1.21 & 2.47 & 4.07 \\
\hline & Melatonin Degradation I & 0 & 2.76 & 4.05 \\
\hline & Ethanol Degradation IV & 1.17 & 2.39 & 3.95 \\
\hline & Tryptophan Degradation X (Mammalian, via Tryptamine) & 1.17 & 2.39 & 3.95 \\
\hline & Superpathway of Melatonin Degradation & 0 & 2.65 & 3.9 \\
\hline & Nicotine Degradation II & 0.76 & 2.63 & 3.88 \\
\hline & Bupropion Degradation & 0 & 2.31 & 3.84 \\
\hline & Serotonin Degradation & 0.74 & 2.56 & 3.77 \\
\hline & Acetone Degradation I (to Methylglyoxal) & 0 & 2.25 & 3.74 \\
\hline & Dopamine Degradation & 1.09 & 2.22 & 3.69 \\
\hline & Retinoate Biosynthesis I & 1.02 & 3.46 & 2.09 \\
\hline & Ethanol Degradation II & 1 & 2.03 & 3.4 \\
\hline & Lanosterol Biosynthesis & 0 & 2.38 & 2.39 \\
\hline
\end{tabular}

Values represent $-\log _{10}$ of the p-values calculated using IPA (Fisher's exact test). Statistically significant changes are indicated by grey shading. 
Mechanism-based quantitative toxicogenomic analysis of cholestasis

effects related to oxidative stress (GADD45 signaling, glutathione-mediated detoxification, NRF2-mediated oxidative stress response). These results indicated that toxicogenomics could discriminate based on the cholestatic mechanism of action.

\section{Quantitative analysis identified good agreement between aPoD and tPoD}

The aPoD for each chemical is summarized in Table 3. Whereas six of the 12 test compounds (CSA, FT, MTS, ANIT, SUL and TMX) showed increased cholestatic markers, the other six (CPZ, DFNa, EME, IMI, KC and $\mathrm{PhB}$ ) did not. To simplify the comparison of aPoD and tPoD, we analyzed these compounds separately.

For the six chemicals that produced increased cholestatic markers, the correlations between $\mathrm{aPoD}$ and $\mathrm{tPoD}$ are shown by scatter plots in Fig. 4 (A and B). There were no significant differences between tPoD and $\mathrm{aPoD}$, and both $\mathrm{tBMD}$ and tBMDL highly correlated with $\mathrm{aPoD}$ (tBMD, $\mathrm{R}=0.73$; tBMDL, $\mathrm{R}=0.77$ ). For the remaining six chemicals that did not produce increased cholestatic markers, the correlations between aPoD and tPoD are shown by scatter plots in Fig. 4 (C and D). tPoD values were significantly lower than aPoD values, and both tBMD and $\mathrm{tBMDL}$ were very highly correlated with aPoD (tBMD, $\mathrm{R}=0.99$; tBMDL, $\mathrm{R}=0.99$ ). In particular, the ratio of tBMDL values to $\mathrm{aPoD}$ values of these 12 chemicals were within 6-fold.

\section{DISCUSSION}

The present study aimed to develop a practical tool for cholestatic DILI risk assessment using qualitative and quantitative toxicogenomics methods, which combined unsupervised analyses and tBMD. This study resulted in two major conclusions. Firstly, known cholestatic compounds were classified into three major groups using qualitative analyses and secondly, we found that the tPoD derived from the differentially expressed gene dose-response could predict aPoD.

DILI represents a major challenge for risk assessment during the development of pharmaceuticals and other chemical substances, such as cosmetic ingredients (Vinken et al., 2012). The range of potential mechanism of action involved means that DILI is not always detected by canonical methods. Thus, the present study indicated a novel qualitative and quantitative DILI assessment based on the mechanism of action.

The present mechanistic classification of 12 compounds by combined unsupervised analysis identified three mechanism of action groups. Our use of a combination of PCA and cluster analysis was shown to be useful for this classification. Although the application of PCA alone could also reveal the relationship between substance groups (Watanabe et al., 2012; Furihata et al., 2016), this approach requires expert judgment to achieve accurate classification. On the other hand, use of cluster analysis alone can discriminate substance groups without expert judgmente; however, it often produces less separation of the sub-groups such as was observed for Groups 2 and 3. This sub-group classification was considered very important in the present study, since canonical pathway analysis identified Group 2- and 3-specific pathways. Therefore, the classification into three groups that was achieved

Table 3. Summary of aPoD for each test compound.

\begin{tabular}{|c|c|c|c|c|c|c|c|c|c|c|c|}
\hline \multirow[b]{2}{*}{ Name } & \multicolumn{3}{|c|}{ ALP } & \multicolumn{3}{|c|}{ Bilirubin } & \multicolumn{3}{|c|}{ GTP } & \multicolumn{2}{|c|}{ aPoD } \\
\hline & Low & Middle & High & Low & Middle & High & Low & Middle & High & $\begin{array}{l}\text { Cholestatic } \\
\text { NOAEL } \\
(\mathrm{mg} / \mathrm{kg} / \text { day })\end{array}$ & $\begin{array}{c}\text { NOAEL without } \\
\text { cholestatic markers } \\
(\mathrm{mg} / \mathrm{kg} / \text { day })\end{array}$ \\
\hline ANIT & - & - & + & - & - & + & - & - & + & 5 & N.D. \\
\hline CSA & - & + & + & - & + & + & - & - & - & 10 & N.D. \\
\hline FT & - & - & - & - & - & + & - & - & - & 50 & N.D. \\
\hline MTS & - & - & - & - & - & + & - & - & + & 100 & N.D. \\
\hline SUL & - & - & - & - & - & - & - & - & + & 15 & N.D. \\
\hline TMX & - & + & + & - & - & - & - & - & - & 6 & N.D. \\
\hline CPZ & - & - & - & - & - & - & - & - & - & N.D. & 45 \\
\hline DFNa & - & - & - & - & - & - & - & - & - & N.D. & 10 \\
\hline EME & - & - & - & - & - & - & - & - & - & N.D. & 1,000 \\
\hline IMI & - & - & - & - & - & - & - & - & - & N.D. & 100 \\
\hline $\mathrm{KC}$ & - & - & - & - & - & - & - & - & - & N.D. & 100 \\
\hline $\mathrm{PhB}$ & - & - & - & - & - & - & - & - & - & N.D. & 200 \\
\hline
\end{tabular}

N.D., not determined; +, increased level; -, unchanged level. 
T. Kawamoto et al.

(A)

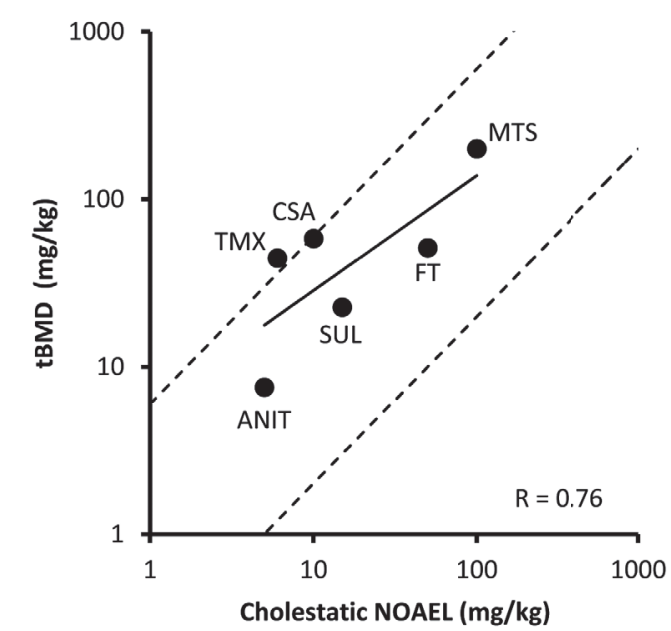

(C)

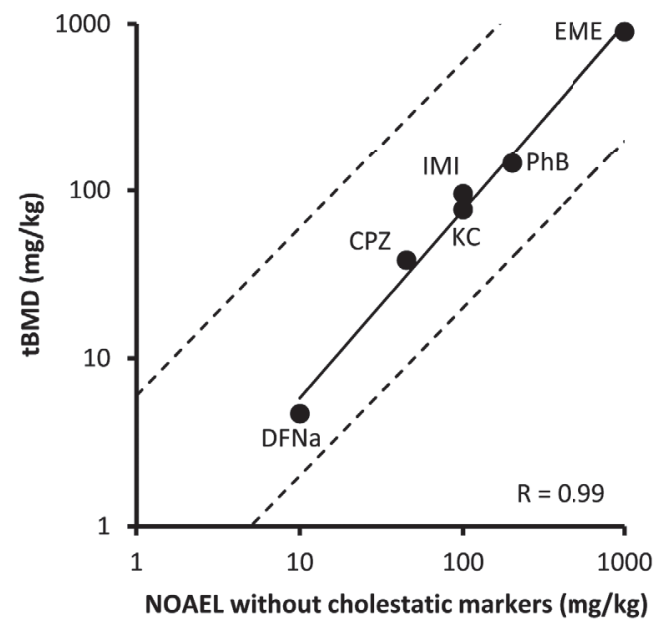

(B)

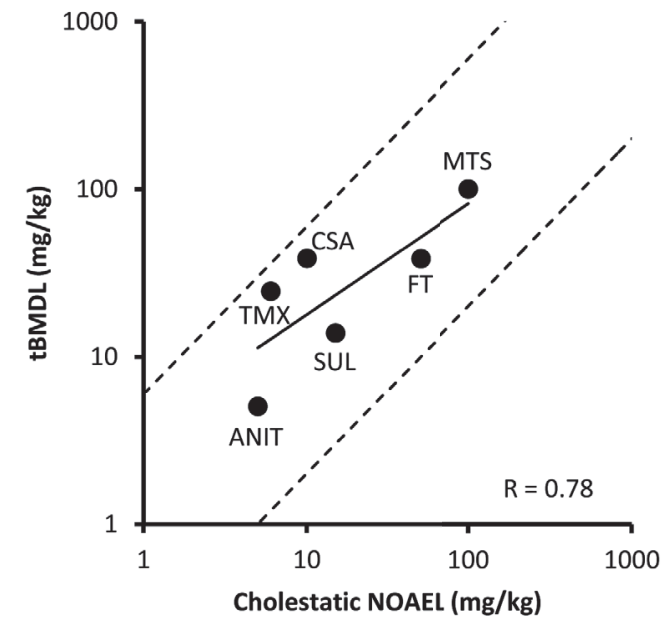

(D)

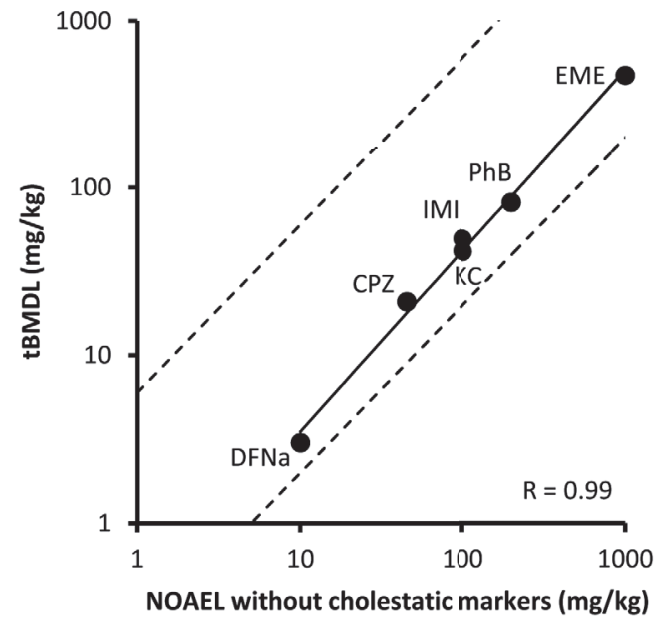

Fig. 4. Correlation between $\mathrm{tPoD}$ and $\mathrm{aPoD}$ for six cholestatic chemicals. tBMD or tBMDL were plotted with aPoD as scatter plots, as indicated. (A) tBMD with cholestatic NOAEL. (B) tBMDL with cholestatic NOAEL. (C) tBMD with NOAEL without cholestatic markers (D) tBMDL with NOAEL without cholestatic markers. $\mathrm{R}=$ correlation coefficient, dashed line $=$ factor 6 .

by the combination of PCA and cluster analysis would be appropriate for $\mathrm{tPoD}$ calculation.

Pathway analyses identified three major mechanisms related to cholestasis. A previous study had reported that the inflammatory response, highlighted by Group 1 compounds in the present study, could cause cholestasis through indirect inhibition of BSEP (Hartmann et al., 2002). The lipid metabolism pathway highlighted by Group 2 (Table 2) could be associated with bilirubin upregulation (Hirode et al., 2009). In addition, cholestasis has been reported to contribute to dysfunction of cholesterol biosynthesis (Nemes et al., 2016). The nuclear receptors identified by Group 3 suggested that CAR and PXR were associated with cholestasis. These receptors are key molecules for exogenous xenobiotic metabolism and endogenous lipid metabolism during adaptive and physiological responses. Chronic dysregulation of these receptors, which include the bile acid sensor, farnesoid $\mathrm{X}$ receptor, may be involved in the etiology of cholestasis. Some studies analyzed CSA-induced cholestatic dif- 
Mechanism-based quantitative toxicogenomic analysis of cholestasis

ferentially expressed genes in vivo (Driessen et al., 2015) and in vitro (Van den Hof et al., 2014b, 2015) and reported similar findings relating to lipid metabolism and nuclear receptor activation. It should be mentioned that the cholestasis-related gene, Scd1 (Flowers et al., 2006), was also included in this group. These previous findings were therefore consistent with the three major mechanisms identified in the present study. Non-cholestatic chemicals were not evaluated in the present study and the identified differentially expressed genes might not therefore represent cholestasis specific changes. Further screening of genes would thus be necessary to elucidate the specific mechanisms of action of cholestatic chemicals.

The present study calculated tPoD values using each differentially expressed gene set within the different mechanism of action groups. With regards to the difference between tPoD and aPoD, tPoD values (median values of $\mathrm{tBMD}$ or $\mathrm{tBMDL}$ ) were significantly lower than $\mathrm{aPoD}$ for chemicals that did not increase cholestatic markers (Fig. 4C, 4D), whereas there were no significant differences between $\mathrm{TPOD}$ and $\mathrm{aPoD}$ for chemicals that altered cholestatic markers (Fig. 4A, 4B). These tPoD values correlated closely with $\mathrm{aPoD}$ and the ratio of tBMDL values to $\mathrm{aPoD}$ values of these chemicals were within 6-fold. Thus, it would be better to use tBMDL to provide a conservative assessment. This result indicated that tPoD could be used to achieve quantitative assessment of DILI risk; this was consistent with a previous report by Thomas et al. (2013) describing the usefulness of tPoD for chemical risk assessment. However, the comparison of tPoD with aPoD in the present study was conducted using 28-days repeated toxicity test data. Thus, further studies should be performed to compare tPoD and aPoD values determined by chronic toxicity studies.

In conclusion, our findings have provided objective qualitative mechanism of action classification methods that could facilitate identification of more accurate toxicity markers. Furthermore, tPoD values can be used to assess cholestatic potential quantitatively, even if aPoD is not derived. In addition, our methodology has a major advantage over canonical binary (positive or negative) toxicogenomics methodology and could provide an alternative approach to assessing drugs during the development process. Further validation studies using an external dataset should be conducted using this approach to examine compounds associated with cholestasis and other DILI including cirrhosis, fibrosis, and lipidosis/phospholipidosis.

\section{ACKNOWLEDGMENTS}

Funding for the present research was provided by Kao
Corporation.

Conflict of interest---- The authors declare that there is no conflict of interest.

\section{REFERENCES}

Benjamin, S.B., Ishak, K.G., Zimmerman, H.J. and Grushka, A. (1981): Phenylbutazone liver injury: a clinical-pathologic survey of 23 cases and review of the literature. Hepatology, 1, 255-263.

Björnsson, E. and Olsson, R. (2005): Outcome and prognostic markers in severe drug-induced liver disease. Hepatology, 42, 481-489.

Boverhof, D.R. and Zacharewski, T.R. (2006): Toxicogenomics in risk assessment: applications and needs. Toxicol. Sci., 89, 352360 .

Chepelev, N.L., Moffat, I.D., Labib, S., Bourdon-Lacombe, J., Kuo, B., Buick, J.K., Lemieux, F., Malik, A.I., Halappanavar, S., Williams, A. and Yauk, C.L. (2015): Integrating toxicogenomics into human health risk assessment: lessons learned from the benzo[a]pyrene case study. Crit. Rev. Toxicol., 45, 44-52.

Clewell, H.J., Efremenko, A., Campbell, J.L., Dodd, D.E. and Thomas, R.S. (2014): Transcriptional responses in the rat nasal epithelium following subchronic inhalation of naphthalene vapor. Toxicol. Appl. Pharmacol., 280, 78-85.

Diehl, A.M., Latham, P., Boitnott, J.K., Mann, J. and Maddrey, W.C. (1984): Cholestatic hepatitis from erythromycin ethylsuccinate report of two cases. Am. J. Med., 76, 931-934.

Dong, H., Gill, S., Curran, I.H., Williams, A., Kuo, B., Wade, M.G. and Yauk, C.L. (2016): Toxicogenomic assessment of liver responses following subchronic exposure to furan in Fischer F344 rats. Arch. Toxicol., 90, 1351-1367.

Driessen, M., Vitins, A.P., Pennings, J.L., Kienhuis, A.S., Water, B.V. and van der Ven, L.T. (2015): A transcriptomics-based hepatotoxicity comparison between the zebrafish embryo and established human and rodent in vitro and in vivo models using cyclosporine A, amiodarone and acetaminophen. Toxicol. Lett., 232, 403-412.

Fattinger, K., Funk, C., Pantze, M., Weber, C., Reichen, J., Stieger, B. and Meier, P.J. (2001): The endothelin antagonist bosentan inhibits the canalicular bile salt export pump: A potential mechanism for hepatic adverse reactions. Clin. Pharmacol. Ther., 69, 223-231.

Ferreira, F.M., Oliveira, P.J., Rolo, A.P., Santos, M.S., Moreno, A.J., da Cunha, M.F., Seiça, R. and Palmeira, C.M. (2003): Cholestasis induced by chronic treatment with alpha-naphthylisothiocyanate (ANIT) affects rat renal mitochondrial bioenergetics. Arch. Toxicol., 77, 194-200.

Flowers, M.T., Groen, A.K., Oler, A.T., Keller, M.P., Choi, Y., Schueler, K.L., Richards, O.C., Lan, H., Miyazaki, M., Kuipers, F., Kendziorski, C.M., Ntambi, J.M. and Attie A.D. (2006): Cholestasis and hypercholesterolemia in SCD1-deficient mice fed a low-fat, high-carbohydrate diet. J. Lipid Res., 47, 26682680.

Fujii, T. (1997): Toxicological correlation between changes in blood biochemical parameters and liver histopathological findings. J. Toxicol. Sci., 22, 161-183.

Funk, C., Pantze, M., Jehle, L., Ponelle, C., Scheuermann, G., Lazendic, M. and Gasser, R. (2001): Troglitazone-induced intrahepatic cholestasis by an interference with the hepatobiliary export of bile acids in male and female rats. Correlation with the 
gender difference in troglitazone sulfate formation and the inhibition of the canalicular bile salt export pump (Bsep) by troglitazone and troglitazone sulfate. Toxicology, 167, 83-98.

Furihata, C., Watanabe, T., Suzuki, T., Hamada, S. and Nakajima, M. (2016): Collaborative studies in toxicogenomics in rodent liver in JEMS.MMS; a useful application of principal component analysis on toxicogenomics. Genes Environ., 38, 1-15.

Gautier, L., Cope, L., Bolstad, B.M. and Irizarry, R.A. (2004): affy-analysis of Affymetrix GeneChip data at the probe level. Bioinformatics, 20, 307-315.

Gusenleitner, D., Auerbach, S.S., Melia, T., Gómez, H.F., Sherr, D.H. and Monti, S. (2014): Genomic models of short-term exposure accurately predict long-term chemical carcinogenicity and identify putative mechanisms of action. PLoS One, 9, 1-15.

Hartmann, G., Cheung, A.K. and Piquette-Miller, M. (2002): Inflammatory cytokines, but not bile acids, regulate expression of murine hepatic anion transporters in endotoxemia. J. Pharmacol. Exp. Ther., 303, 273-281.

Hirode, M., Horinouchi, M., Uehara, T., Ono, A., Miyagishima, T., Yamada, H., Nagao, T., Ohno, Y. and Urushidani, T. (2009): Gene expression profiling in rat liver treated with compounds inducing elevation of bilirubin. Hum. Exp. Toxicol., 28, 231244.

Hong, F., Breitling, R., McEntee, C.W., Wittner, B.S., Nemhauser, J.L. and Chory, J. (2006): RankProd: A bioconductor package for detecting differentially expressed genes in meta-analysis. Bioinformatics, 22, 2825-2827.

Igarashi, Y., Nakatsu, N., Yamashita, T., Ono, A., Ohno, Y., Urushidani, T. and Yamada, H. (2015): Open TG-GATEs: A large-scale toxicogenomics database. Nucleic Acids Res., 43, D921-D927.

Krämer, A., Green, J., Pollard, J.Jr. and Tugendreich, S. (2014): Causal analysis approaches in Ingenuity Pathway Analysis. Bioinformatics, 30, 523-530.

Liu, S., Kawamoto, T., Morita, O., Yoshinari, K. and Honda, H. (2017): Discriminating between adaptive and carcinogenic liver hypertrophy in rat studies using logistic ridge regression analysis of toxicogenomic data: The mode of action and predictive models. Toxicol. Appl. Pharmacol., 318, 79-87.

Matsumoto, H., Saito, F. and Takeyoshi, M. (2014): CARCINO-

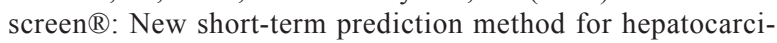
nogenicity of chemicals based on hepatic transcript profiling in rats. J. Toxicol. Sci., 39, 725-734.

Morgan, R.E., Trauner, M., van Staden, C.J., Lee, P.H., Ramachandran, B., Eschenberg, M., Afshari, C.A., Qualls, C.W.Jr., Lightfoot-Dunn, R. and Hamadeh, H.K. (2010): Interference with bile salt export pump function is a susceptibility factor for human liver injury in drug development. Toxicol. Sci., $118,485-500$.

National Research Council (US) Committee on Applications of Toxicogenomic Technologies to Predictive Toxicology. (2007): Applications of Toxicogenomic Technologies to Predictive Toxicology and Risk Assessment.

Nemes, K., Åberg, F., Gylling, H. and Isoniemi, H. (2016): Cholesterol metabolism in cholestatic liver disease and liver transplantation: From molecular mechanisms to clinical implications. World J. Hepatol., 8, 924-932.

Organisation for Economic Co-operation and Development. (2016): Users' Handbook supplement to the Guidance Document for developing and assessing Adverse Outcome Pathways.

Ogimura, E., Sekine, S. and Horie, T. (2011): Bile salt export pump inhibitors are associated with bile acid-dependent drug-induced toxicity in sandwich-cultured hepatocytes. Biochem. Biophys.
Res. Commun., 416, 313-317.

Olson, H., Betton, G., Robinson, D., Thomas, K., Monro, A., Kolaja, G., Lilly, P., Sanders, J., Sipes, G., Bracken, W., Dorato, M., Van Deun, K., Smith, P., Berger, B. and Heller, A. (2000): Concordance of the toxicity of pharmaceuticals in humans and in animals. Regul. Toxicol. Pharmacol., 32, 56-67.

Ozer, J., Ratner, M., Shaw, M., Bailey, W. and Schomaker, S. (2008): The current state of serum biomarkers of hepatotoxicity. Toxicology, 245, 194-205.

Scientific Committee on Consumer Safety NfG (2016): The SCCS notes of guidance for the testing of cosmetic ingredients and their safety evaluation. SCCS/1564/15.

Padda, M.S., Sanchez, M., Akhtar, A.J. and Boyer, J.L. (2011): Drug-induced cholestasis. Hepatology, 53, 1377-1387.

Suzuki, R. and Shimodaira, H. (2006): Pvclust: An R package for assessing the uncertainty in hierarchical clustering. Bioinformatics, 22, 1540-1542.

Thomas, R.S., Wesselkamper, S.C., Wang, N.C., Zhao, Q.J., Petersen, D.D., Lambert, J.C., Cote, I., Yang, L., Healy, E., Black, M.B., Clewell, H.J.3rd., Allen, B.C. and Andersen, M.E. (2013): Temporal concordance between apical and transcriptional points of departure for chemical risk assessment. Toxicol. Sci., 134, 180-194.

Van den Hof, W.F., Coonen, M.L., van Herwijnen, M., Brauers, K., Wodzig, W.K., van Delft, J.H. and Kleinjans, J.C. (2014a): Classification of hepatotoxicants using HepG2 cells: A proof of principle study. Chem. Res. Toxicol., 27, 433-442.

Van den Hof, W.F., Van Summeren, A., Lommen, A., Coonen, M.L., Brauers, K., van Herwijnen, M., Wodzig, W.K. and Kleinjans, J.C. (2014b): Integrative cross-omics analysis in primary mouse hepatocytes unravels mechanisms of cyclosporin A-induced hepatotoxicity. Toxicology, 324, 18-26.

Van den Hof, W.F., Ruiz-Aracama, A., Van Summeren, A., Jennen, D.G., Gaj, S., Coonen, M.L., Brauers, K., Wodzig, W.K., van Delft, J.H. and Kleinjans, J.C. (2015): Integrating multiple omics to unravel mechanisms of Cyclosporin A induced hepatotoxicity in vitro. Toxicol. In Vitro, 29, 489-501.

Vinken, M., Pauwels, M, Ates, G., Vivier, M., Vanhaecke, T. and Rogiers, V. (2012): Screening of repeated dose toxicity data present in SCC(NF)P/SCCS safety evaluations of cosmetic ingredients. Arch. Toxicol., 86, 405-412.

Vinken, M., Landesmann, B., Goumenou, M., Vinken, S., Shah, I., Jaeschke, H., Willett, C., Whelan, M. and Rogiers. V. (2013): Development of an adverse outcome pathway from drug-mediated bile salt export pump inhibition to cholestatic liver injury. Toxicol. Sci., 136, 97-106.

Wang, Y., Lin, Z., Liu, Z., Harris, S., Kelly, R., Zhang, J., Ge, W., Chen, M., Borlak, J. and Tong, W. (2013): A unifying ontology to integrate histological and clinical observations for drug-induced liver injury. Am. J. Pathol., 182, 1180-1187.

Watanabe, T., Suzuki, T., Natsume, M., Nakajima, M., Narumi, K., Hamada, S., Sakuma, T., Koeda, A., Oshida, K., Miyamoto, Y., Maeda, A., Hirayama, M., Sanada, H., Honda, H., Ohyama, W., Okada, E., Fujiishi, Y., Sutou, S., Tadakuma, A., Ishikawa, Y., Kido, M., Minamiguchi, R., Hanahara, I., and Furihata, C. (2012): Discrimination of genotoxic and non-genotoxic hepatocarcinogens by statistical analysis based on gene expression profiling in the mouse liver as determined by quantitative real-time PCR. Mutat. Res., 747, 164-175.

Yang, L., Allen, B.C. and Thomas, R.S. (2007): BMDExpress: a software tool for the benchmark dose analyses of genomic data. BMC Genomics, 8, 387. 